\title{
Dominio del fonoaudiólogo para la determinación del grado de viscosidad de alimentos líquidos
}

\author{
Speech Pathologists Proficiency for Determining \\ Viscosity Grade in Liquid Foods
}

Rodrigo Tobar F.
Fonoaudiólogo
Universidad de Chile

\section{Constanza Campos T.}

Lic. en Fonoaudiología Universidad de Chile

\section{Valentina Cancino $\mathbf{H}$.}

Lic. en Fonoaudiología

Universidad de Chile

\section{Monserrat Diez De Medina E. \\ Lic. en Fonoaudiología Universidad de Chile \\ Nicole Fierro A. \\ Lic. en Fonoaudiología Universidad de Chile}

Contacto con el autor: Rodrigo Tobar $\mathrm{F}$.

Santiago - Chile

Correo-e: rodrigotobar@med.uchile.cl

Recibido: $19 / 03 / 2016$

Aceptado: 22/07/2016

\begin{abstract}
RESUMEN
La disfagia es una alteración del proceso deglutorio que afecta la seguridad, eficacia y calidad de la alimentación. Una de las estrategias más utilizadas para su intervención es la modificación de la viscosidad de los alimentos, sin embargo, la metodología empleada para determinarla es subjetiva y no está estandarizada. Esta investigación buscó establecer el dominio de los fonoaudiólogos para determinar el tipo de viscosidad. Se utilizaron 12 muestras de alimentos líquidos, cuyas viscosidades fueron determinadas objetivamente con un viscosímetro rotacional, clasificándolos bajo las categorías: fino, néctar, miel y pudín. Posteriormente, cuarenta fonoaudiólogos que se desempeñan en el área de los trastornos de la deglución en centros de salud de la Región Metropolitana en Santiago de Chile, evaluaron subjetivamente las 12 muestras. Con esta información se realizó un estudio comparativo objetivo/subjetivo para establecer el dominio de los profesionales. Los participantes lograron $66,87 \%$ de efectividad en la valoración del grado de viscosidad de las muestras, con un mejor rendimiento para aquellas viscosidades tipo fino y pudín. La repetibilidad intrasujeto fue superior a $75 \%$ para el $60 \%$ de la muestra. Existen además indicadores de que el tiempo de ejercicio profesional incidiría positivamente en estas capacidades, no así el nivel de perfeccionamiento. Por último, se establece que los fonoaudiólogos evaluados poseen un dominio regular para determinar el grado de viscosidad de alimentos líquidos, pero este no es homogéneo. Se hace necesario incentivar el conocimiento y manejo adecuado en este tema, en búsqueda de consensuar procedimientos y criterios que permitan una mayor estandarización al respecto.
\end{abstract}

Palabras clave: alimentos, disfagia, fonoaudiólogo, reología, viscosidad.

\begin{abstract}
Dysphagia is a disruption in the swallowing process which hinders movement of food, affecting the safety, efficiency and quality of feeding. Treatment includes different strategies, with viscosity modification being one of the most used strategies nowadays. However, the methodology used to determine food viscosity is subjective and not standardized. In this regard, this study seeks to establish the speech pathologists' skills in determining the different types of food viscosity. Twelve samples of liquid foods were used, whose viscosities were determined objectively with a rotational viscometer. Then, the samples of liquid foods were classified into four categories: thin liquid, nectar, honey, and pudding. Forty speech pathologists working with patients diagnosed with dysphagia at health centers in the Metropolitan Region, Chile, subjectively evaluated the samples of liquid foods. Finally, an objective/subjective comparative study was performed to determine their competence in identifying the different types of food viscosity. Near $66,87 \%$ of the participants performed well in the task. Participants performed better in determining thin and pudding viscosities. Over $75 \%$ of intra-subject repeatability was obtained for $60 \%$ of the sample. Unlike postgraduate studies, it could be observed that professional experience has a positive impact on these skills. Finally, the results of this study indicate that the participants have intermediate skills in determining the degree of viscosity of liquid food. However, this is not homogeneous, and therefore, it is necessary to enhance our understanding and proper management of dysphagia. Agreement on standard procedures is also necessary.
\end{abstract}

Keywords: dysphagia, food, speech pathologist, reology, viscosity. 


\section{Introducción}

\section{Alimentación y disfagia}

La alimentación es una actividad trascendental para la sobrevida humana y su ejecución involucra una serie de aspectos socioemocionales, por lo que su indemnidad es un componente esencial para el bienestar psicológico de cada individuo. Actualmente, los trastornos que implican alguna anomalía en la realización de esta actividad son cada vez más usuales en la población adulta, siendo la disfagia uno de los más frecuentes (Tobar, 2010).

En la literatura existen muchas definiciones de disfagia, pero en términos generales, se describe como una alteración del proceso deglutorio, en una o más de sus fases, lo que se traduce en una dificultad en el desplazamiento del alimento desde la cavidad oral hasta el estómago (Clavé et al., 2006; Logemann, 1998). Por su parte, O'Leary, Hanson y Smith (2010) definen la disfagia como una situación de salud donde se describe una serie de desórdenes mecánicos que afectan la seguridad, la eficacia y la calidad de la alimentación.

De acuerdo con la fase del proceso deglutorio que se encuentre alterada, la disfagia se puede clasificar en dos tipos: disfagia orofaríngea y disfagia esofágica. La primera incluye las fases anticipatoria, preparatoria oral, oral y faríngea; mientras que la segunda comprende solo la fase esofágica (González y Bevilacqua, 2009; Logemann, 1998; Matsuo \& Palmer, 2009; Nazar, Ortega, Godoy, Godoy y Fuentealba, 2008).

\section{Consecuencias y severidad de la disfagia}

Al encontrarse seriamente comprometida la capacidad y el control sensoriomotor en la disfagia, una de las principales complicaciones es la aspiración pulmonar. Esta última origina gran preocupación por el riesgo asociado a la obstrucción de la vía aérea, así como por la generación de neumonías y/o fallas respiratorias, las que ponen en riesgo la vida del individuo. Además, pueden presentarse otras complicaciones como deshidratación, malnutrición e incluso la muerte (Clavé et al., 2008; O’Leary, et al., 2010; Perry \& Love, 2001).

Al considerar las complicaciones propias de la disfagia, se establecen distintos niveles de severidad de acuerdo con el grado de afectación de las estructuras implicadas en la deglución y las recomendaciones del tipo de ingesta oral necesario para garantizar una deglución segura.

\section{Contexto de intervención terapéutica en la alimentación de personas con disfagia}

Dentro del contexto terapéutico se sugiere, como una estrategia eficiente para disminuir las complicaciones asociadas a este trastorno, efectuar modificaciones alimenticias para distintas condiciones deglutorias (Clavé et al., 2006; Goulding \& Bakheit, 2000; Paik et al., 2004), con el fin de que aquellos usuarios aptos para una alimentación por vía oral, la realicen de forma segura, manteniendo una adecuada hidratación y nutrición.

Aplicar cambios a las propiedades físicas de los alimentos es una de las recomendaciones más difundidas en el tratamiento fonoaudiológico de 
Tobar, R., Campos, C., Cancino, V., Diez De Medina, M. y Fierro, N.

usuarios con disfagia. La estrategia más utilizada corresponde a la manipulación de consistencias, aunque su aplicación como única variable resulta insuficiente para dar respuesta a las necesidades de cada usuario, debido a que solo se basa en el grado de cohesión y compresibilidad de los alimentos. Considerar, además, otras propiedades como la viscosidad permite clasificar con mayor precisión los alimentos (Tobar, 2010).

En relación con esto, se propone el análisis de otras propiedades dentro de las que destaca la viscosidad de los alimentos, especialmente para los de consistencia líquida.

El concepto de viscosidad corresponde a una unidad de medida objetiva, valorada en centipoise (cP) y requiere de la utilización de un viscosímetro para su determinación (National Dysphagia Task Force, American Dietetic Association, 2002).

\section{Viscosidad y deglución de alimentos líquidos}

La viscosidad afecta significativamente el desplazamiento de un alimento líquido durante la deglución, dado que influye en el tiempo de tránsito del bolo, en la apertura cricofaríngea y en la eficiencia de la deglución orofaríngea (Hanson, O'Leary y Smith, 2012). Sobre la base del modo de desplazamiento de distintos fluidos, Penman y Thomson (1998) realizaron una clasificación de los alimentos líquidos que se ajusta a las necesidades de los pacientes con trastornos de la deglución, la cual se detalla en la Tabla 1.

Tabla 1

Clasificación de la viscosidad de los fluidos (Penman \& Thompson, 1998)

\begin{tabular}{lcc}
\hline \multicolumn{1}{c}{ Escala de alimentos } & $\begin{array}{c}\text { Descripción de la viscosidad de los líquidos } \\
\text { (cP) }\end{array}$ & $\begin{array}{c}\text { Valores de viscosidad en centipoise } \\
\text { Líquido fino }\end{array}$ \\
Néctar & $\begin{array}{c}\text { Agua y todos los jugos más delgados que el jugo } \\
\text { de manzana }\end{array}$ & $51-350 \mathrm{cP}$ \\
Miel & Líquidos con viscosidad del néctar & $351-1.750 \mathrm{cP}$ \\
Pudín & Líquidos con viscosidad de la miel & 1.750 o más cP \\
\hline
\end{tabular}

Considerando lo anterior, la viscosidad es un elemento importante en la intervención de personas con disfagia, ya que los alimentos líquidos se comportan de manera distinta al momento de ser introducidos en la cavidad oral (Hanson et al., 2012), lo cual obedece a las características que tienen estos alimentos para fluir.
De acuerdo con lo expuesto, considerar la viscosidad en la intervención nutricional de los usuarios con disfagia resulta beneficioso para disminuir los riesgos asociados a las alteraciones deglutorias y mantener, en mayor medida, la variedad alimenticia (Glassburn \& Deem, 1998). 
Los beneficios de la modificación de la viscosidad fueron evidenciados por Clavé et al. (2006) quienes observaron que, en usuarios con disfagia neurogénica, el aumento de la viscosidad producía un incremento significativo de la eficacia y seguridad de la deglución, especialmente con la viscosidad pudín, minimizando las penetraciones y aspiraciones. Por lo tanto, el uso de este grado de viscosidad reduce los índices de neumonía aspirativa e incrementa la cantidad total de agua y calorías que se ingieren en condiciones seguras, mejorando la hidratación y el estado nutricional del usuario.

\section{Viscosidad de los alimentos y práctica clínica}

Las modificaciones físicas en los alimentos líquidos se realizan a partir del criterio clínico de cada fonoaudiólogo. Así, cuando estos deben reproducir cierto grado de viscosidad o seleccionar un producto preparado envasado cuyas etiquetas no informan acerca de la viscosidad, el clínico debe inferir esa información (Steele, Goff \& Van Lieshout, 2003).

Es evidente, entonces, que la selección, preparación y administración de la viscosidad de los alimentos líquidos, continúa siendo un procedimiento subjetivo (Ekberg, 2012). Por consiguiente, si no se cuenta con las aptitudes para reproducir de forma fiable las viscosidades, los usuarios con disfagia no obtienen beneficios clínicos acorde con sus necesidades.

Desafortunadamente, los profesionales responsables de la prescripción de una dieta modificada sobre la base de la viscosidad han demostrado tener un conocimiento limitado en este campo, tal como se evidenció en la investigación de Glassburn y Deem (1998), quienes examinaron la variabilidad entre fonoaudiólogos de Estados Unidos al mezclar consistencias líquidas espesadas para la evaluación y manejo de la disfagia; los resultados indicaron que no existía ninguna relación o repetibilidad entre ellos en sus intentos de mezclas de miel y néctar; sin embargo, cabe destacar que las variaciones intrasujeto fueron algo más repetibles.

De los resultados anteriores se desprende que el juicio subjetivo de la viscosidad no es un método confiable, ya que pese a que los sujetos mostraron repetibilidad a nivel individual, los niveles de viscosidad no se encuentran estandarizados, por lo que no existe consenso al respecto entre los profesionales. En consecuencia, es importante objetivar esta propiedad, de manera que los fonoaudiólogos cuenten con la misma línea de base para adquirir un dominio adecuado del tema (Clavé et al., 2006; Glassburn \& Deem, 1998).

Bajo esta premisa, actualmente existen normas adecuadas a cada país en la indicación de una determinada dieta para usuarios con disfagia, dentro de las cuales destacan las de Estados Unidos, Reino Unido y Australia, quienes han incorporado mediciones objetivas para la clasificación de alimentos y líquidos, según textura y viscosidad (Atherton Bellis-Smith, Cichero \& Suter, 2007). Sin embargo, en el contexto nacional, a diferencia de estos países, aún no existe una estandarización respecto de la clasificación y metodología utilizada para determinar el grado de viscosidad de los alimentos líquidos (Tobar, 2010). 
Es evidente, entonces, que para reducir la probabilidad de eventos adversos, es necesario lograr un consenso entre profesionales fonoaudiólogos chilenos acerca de la modificación de la viscosidad de los alimentos líquidos administrados a usuarios con alteraciones en la deglución. Además, cabe resaltar la importancia de establecer un lenguaje común para reducir errores en la selección de productos alimenticios, tanto en centros de salud como en los hogares de los usuarios que se encuentran en rehabilitación. De acuerdo con esto, una estandarización en los criterios para determinar el grado de viscosidad, otorgaría una mayor seguridad en el tratamiento de un individuo con disfagia y aseguraría un acceso seguro y pertinente a sus necesidades alimenticias (Atherton et al., 2007).

Ante la situación planteada, el propósito de este estudio contempla obtener el insumo inicial para la elaboración de planes de perfeccionamiento que permitan, a quienes ya se desempeñan en el área de los trastornos de la deglución, consensuar procedimientos y criterios para la determinación del grado de viscosidad de los alimentos utilizados durante el tratamiento de personas con disfagia. Se contempla, además, que esta información pueda implicar la paulatina modificación y actualización de los planes de formación de pregrado, permitiendo entregar mejores herramientas en esta área a los futuros profesionales.
De acuerdo con lo anterior, se busca describir el dominio de un grupo de fonoaudiólogos que se desempeñan en el área de trastornos de la deglución en la Región Metropolitana, para determinar el grado de viscosidad de 12 alimentos líquidos preparados, factibles de utilizar en la intervención de personas con disfagia.

\section{Método}

Se utilizó un diseño de tipo descriptivo, exploratorio y transversal, seleccionándose por ello estadística descriptiva para la posterior presentación de los resultados.

Las variables consideradas en esta investigación fueron las siguientes:

a) Nivel de efectividad de los profesionales fonoaudiólogos en la valoración del grado de viscosidad de 12 líquidos utilizados en el tratamiento de personas con disfagia. Este fue establecido y caracterizado según el número total de aciertos en la determinación, con un máximo de 12 y un mínimo de cero. Por conveniencia y también para facilitar su análisis se estratificó en tres grupos el rendimiento de los sujetos, según se describe en la Tabla 2.

Tabla 2

Grados de efectividad de los profesionales, según cantidad de aciertos en la determinación de viscosidad

\begin{tabular}{cc}
\hline Total de aciertos & Nivel de efectividad \\
\hline $0-4$ & Nivel insuficiente \\
$5-8$ & Nivel regular \\
$9-12$ & Nivel suficiente \\
\hline
\end{tabular}


b) Viscosidad de alimentos líquidos utilizados en el tratamiento de personas con trastornos de la deglución, según la clasificación de Penman y Thomson (1998).

c) Competencia de los profesionales fonoaudiólogos en el reconocimiento de la viscosidad, determinada el porcentaje absoluto de acuerdo con el número de aciertos de todos los profesionales en cada grado de viscosidad.

d) Tiempo de ejercicio profesional en el área de trastornos de la deglución, el cual fue calificado en rango de años desde su inicio hasta la fecha de la entrevista inicial (0 a 4,11 años; 5 a 9,11 años y 10 años o más).

e) Nivel de perfeccionamiento, considerando el grado académico o especialización en el área. Por conveniencia, se asignó un determinado puntaje según naturaleza y duración del curso (magíster, 6 puntos; diploma, 3 puntos; y curso de postítulo, 1 punto) y se estratificó el nivel de perfeccionamiento posteriormente en tres grupos descritos en la Tabla 3.

Tabla 3

Nivel de perfeccionamiento de los profesionales fonoaudiólogos

\begin{tabular}{cc}
\hline Puntaje total cursos de perfeccionamiento & Nivel de perfeccionamiento \\
\hline $0-3$ puntos & Bajo \\
$4-7$ puntos & Medio \\
8 o más puntos & Alto \\
\hline
\end{tabular}

f) Repetibilidad en el nivel de efectividad para la valoración del grado de viscosidad de los 12 alimentos líquidos utilizados en el tratamiento de personas con disfagia.

\section{Participantes}

La muestra estuvo conformada por 40 profesionales fonoaudiólogos seleccionados por conveniencia según dos criterios de inclusión: ejercer laboralmente en la Región Metropolitana (en centros públicos y/o privados) y desempeñarse en el área de trastornos de la deglución (en población infantil y/o adulta).

\section{Procedimientos para la obtención de datos}

Se realizaron tres procedimientos para la obtención de los datos. En primer término, se seleccionaron las muestras de alimentos líquidos. En una instancia previa y a través del uso de un viscosímetro rotacional digital Brookfield DV2T, se realizó una medición objetiva de la viscosidad de un amplio grupo de alimentos líquidos disponibles en el mercado chileno, los que fueron seleccionados de forma aleatoria. Este procedimiento se efectuó en los laboratorios del Departamento de Ciencia y Tecnología Farmacéutica de la Facultad de Ciencias Químicas y Farmacéuticas de la Universidad de Chile.

Posteriormente, de este grupo de líquidos, el equipo de investigación seleccionó por conveniencia 12 de ellos, correspondientes a un líquido tipo fino; dos líquidos tipo néctar; tres líquidos tipo miel; y seis líquidos tipo pudín. La selección anterior se basó en las dificultades demostradas por los fonoaudiólogos para determinar estas viscosidades, tal como lo 
señalado en estudios como el de Glassburn y Deem (1998).

Un segundo procedimiento consistió en la construcción de tres instrumentos de recopilación de datos:

a) Carta de convocatoria a los participantes. La invitación a participar como sujeto de estudio se realizó por correo electrónico, adjuntando un documento que detallaba cada aspecto del trabajo, con el fin de que el interesado contara con información suficiente para aceptar participar en el estudio. A su vez, se les extendió un consentimiento informado, el cual avalaba que el sujeto conocía todos los detalles de la investigación, estaba de acuerdo con ellos y aceptaba el uso y posterior análisis de sus respuestas.

b) Pauta de registro de antecedentes. Instrumento elaborado con el objetivo de recopilar los antecedentes del grupo de estudio.

c) Cartilla de respuestas. Instrumento utilizado por los profesionales fonoaudiólogos para indicar la valoración de la viscosidad que realizaron en cada una de las muestras de alimento líquido.

Por último, como tercer procedimiento se realizó una valoración del grado de viscosidad de los alimentos líquidos seleccionados en cuatro etapas:

a) Durante la primera evaluación se efectuó una reunión presencial con cada participante donde este debía determinar, según su propia técnica, el grado de viscosidad de los alimentos presentados. Para ello, los 12 alimentos líquidos seleccionados anteriormente se trasladaron a un sistema de mantención de temperatura que permitió continuar la línea de frío y conservar de forma homogénea las características de viscosidad de cada muestra.

b) Todos los alimentos se presentaron en formatos iguales, dentro de 12 recipientes plásticos de 200 ml. El orden de presentación de cada una de las muestras fue elegido de manera aleatoria, siendo el mismo para todos los sujetos. Solo el equipo de investigación contaba con dicha información.

c) El fonoaudiólogo debió clasificar cada una de las muestras, según su viscosidad, en una de las siguientes categorías: líquido fino, néctar, miel y pudín; y consignar su elección en la cartilla de respuestas.

d) Transcurridas a lo menos dos semanas, el fonoaudiólogo debió realizar una segunda valoración del grado de viscosidad de los alimentos líquidos seleccionados. Se utilizó el mismo procedimiento, modificando solo el orden de presentación de las muestras.

\section{Resultados}

\section{Descripción de los sujetos en estudio}

A partir de la información recabada en la pauta de registro de antecedentes, cabe destacar que la mayor parte del grupo presenta un tiempo de ejercicio profesional y un nivel de perfeccionamiento bajos, como se observa en la Tabla 4. 
Tabla 4

Caracterización de los profesionales fonoaudiólogos según el tiempo de ejercicio y perfeccionamiento en el área

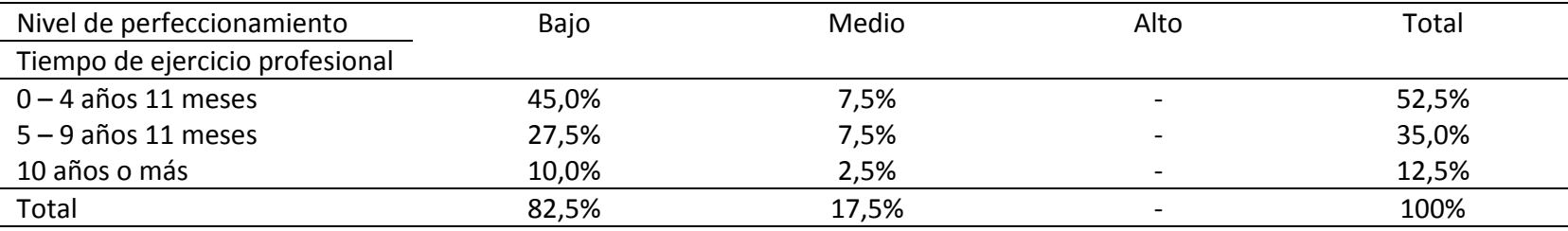

\section{Nivel de efectividad del grupo en estudio}

Respecto del nivel de efectividad promedio del grupo en la valoración del grado de viscosidad de las muestras, se encontró que esta alcanzó un 66,87\%,

Tabla 5

Nivel de efectividad grupal en la valoración del grado de viscosidad

\begin{tabular}{lcc}
\hline Nivel de efectividad grupal en la valoración del grado viscosidad & Promedio de aciertos & Efectividad (\%) \\
\hline Primera medición & 7,95 & $66,25 \%$ \\
Segunda medición & 8,10 & $67,50 \%$ \\
\hline Total & 8,02 & $66,87 \%$ \\
\hline
\end{tabular}

\section{Nivel de efectividad individual}

En relación con el rendimiento individual de los fonoaudiólogos, la mayor parte de ellos alcanzó un lo que equivale a un nivel de efectividad regular. No se evidenciaron diferencias relevantes entre la primera y segunda medición (Tabla 5). nivel de efectividad suficiente en la valoración de la viscosidad, lo cual se evidencia en la Tabla 6.

Tabla 6

Nivel de efectividad individual en la valoración del grado de viscosidad de 12 alimentos líquidos utilizados en el tratamiento de personas con disfagia

\begin{tabular}{lc}
\hline Nivel de efectividad grupal en la valoración del grado viscosidad & Número de fonoaudiólogos \\
\hline Nivel insuficiente & $5,0 \%$ \\
Nivel regular & $40 \%$ \\
Nivel suficiente & $55 \%$ \\
\hline Total & $100 \%$ \\
\hline
\end{tabular}

\section{Repetibilidad del nivel de efectividad intra-sujeto}

En cuanto a la repetibilidad del nivel de efectividad, un $60 \%$ de los sujetos obtuvo un porcentaje superior al $75 \%$ de repetibilidad, lo cual demuestra que la mayoría de los fonoaudiólogos 
Tabla 7

Repetibilidad del nivel de efectividad para la valoración del grado de viscosidad de 12 alimentos líquidos utilizados en el tratamiento de personas con disfagia

\begin{tabular}{lcccc}
\hline Porcentaje repetibilidad & \multicolumn{3}{c}{ Nivel de efectividad } & Sujetos \\
\cline { 2 - 5 } & Nivel insuficiente & Nivel regular & Nivel suficiente & Total \\
\hline $0 \%-24 \%$ & - & - & - & $2,5 \%$ \\
$25 \%-49 \%$ & - & $2,5 \%$ & - & $37,5 \%$ \\
$50 \%-74 \%$ & $2,5 \%$ & $15,0 \%$ & $20,0 \%$ & $60,0 \%$ \\
$75 \%-100 \%$ & $2,5 \%$ & $22,5 \%$ & $35,0 \%$ & $100 \%$ \\
\hline Total & $5 \%$ & $40 \%$ & $55 \%$ & \\
\hline
\end{tabular}

Nivel de efectividad y tiempo de ejercicio profesional

Al considerar el nivel de efectividad en relación con el tiempo de ejercicio profesional, se observó que la mayor parte de los sujetos se concentró en el rango de menor experiencia profesional (0 - 4 años 11 meses) y la mayoría de ellos obtuvo un nivel de efectividad regular, como se observa en la Tabla 8.

Tabla 8

Relación entre el nivel de efectividad y el tiempo de ejercicio profesional en el área de trastornos de la deglución

\begin{tabular}{lcccc}
\hline Nivel de efectividad & Nivel insuficiente & Nivel regular & Nivel suficiente & Total \\
\hline $\begin{array}{l}\text { Tempo de ejercicio } \\
\text { profesional }\end{array}$ & & & & \\
\hline $0-4$ años 11 meses & $2,5 \%$ & $27,5 \%$ & $22,5 \%$ & $52,5 \%$ \\
$5-9$ años 11 meses & $2,5 \%$ & $10,0 \%$ & $22,5 \%$ & $35,0 \%$ \\
10 años o más & - & $2,5 \%$ & $10,0 \%$ & $12,5 \%$ \\
\hline Total & $5,0 \%$ & $40,0 \%$ & $55 \%$ & $100 \%$ \\
\hline
\end{tabular}

Nivel de efectividad y nivel de perfeccionamiento profesional

En cuanto a la relación entre el nivel de efectividad y el de perfeccionamiento, se aprecia que un gran número de sujetos presentó un nivel de perfeccionamiento bajo, grupo en el cual la mayoría obtuvo un nivel de efectividad suficiente. Cabe destacar que ninguno de los participantes alcanzó un nivel de perfeccionamiento alto. La especificación respecto a esta información se detalla a continuación en la Tabla 9.

Tabla 9

Relación entre el nivel de efectividad y el nivel de perfeccionamiento

\begin{tabular}{lcccc}
\hline \multirow{2}{*}{$\begin{array}{l}\text { Nivel de } \\
\text { perfeccionamiento }\end{array}$} & \multicolumn{3}{c}{ Nivel de efectividad } & Sujetos \\
\cline { 2 - 5 } & Nivel insuficiente & Nivel regular & Nivel suficiente & Total \\
\hline Bajo & $2,5 \%$ & $32,5 \%$ & $47,5 \%$ & $82,5 \%$ \\
Medio & $2,5 \%$ & $7,5 \%$ & $7,5 \%$ & $17,5 \%$ \\
Alto & - & - & - & - \\
\hline Total & $5 \%$ & $40 \%$ & $55 \%$ & $100 \%$ \\
\hline
\end{tabular}




\section{Competencia grupal según tipo de líquido estudiado y viscosidad}

En relación con la competencia de los profesionales fonoaudiólogos para reconocer los distintos tipos de viscosidad, se observó que esta fue mayor en la categoría líquido fino. En la Tabla 10 se recopila la información acerca del porcentaje de competencia, junto con el promedio de aciertos para cada tipo de viscosidad.

Tabla 10

Competencia de los profesionales fonoaudiólogos en el reconocimiento de la viscosidad

\begin{tabular}{lcc}
\hline Tipo de viscosidad & Competencia de fonoaudiólogos (\%) & Promedio de aciertos \\
\hline Líquido fino & $80,0 \%$ & 64,0 \\
Néctar & $56,8 \%$ & 45,5 \\
Miel & $62,5 \%$ & 50,0 \\
Pudín & $70,2 \%$ & 56,1 \\
\hline
\end{tabular}

\section{Competencia grupal por producto}

Respecto de la competencia alcanzada por los fonoaudiólogos al determinar el grado de viscosidad de cada producto, ella fue mayor para el flan de chocolate Nestlé $(98,75 \%)$, mientras que la que presentó menor competencia se evidenció en el yogurt batido de piña Soprole $(38,75 \%)$, ambos pertenecientes a la categoría pudín. En la Tabla 11 se presentan los productos con sus respectivas viscosidades objetivas, así como también el promedio de aciertos y el porcentaje de competencia obtenido por los sujetos.

Tabla 11

Competencia de los profesionales fonoaudiólogos en el reconocimiento de la viscosidad por producto

\begin{tabular}{llccc}
\hline \multirow{2}{*}{ Tipo de viscosidad } & \multicolumn{1}{c}{ Producto } & Viscosidad & $\begin{array}{c}\text { Promedio de } \\
\text { aciertos }\end{array}$ & Competencia (\%) \\
& Leche cultivada Next durazno & $39,45 \mathrm{cP}$ & 64 & $80,0 \%$ \\
\hline \multirow{2}{*}{ Néctar } & Yogurt Bliss Nestlé de frutilla & $155 \mathrm{cP}$ & 43 & $53,7 \%$ \\
& Kids Colun yogurt Squeeze batido de frutilla & $169 \mathrm{cP}$ & 48 & $60,0 \%$ \\
\hline \multirow{3}{*}{ Miel } & Yogurt batido Colun de damasco & $1169 \mathrm{cP}$ & 50 & $62,5 \%$ \\
& Compota de durazno Soprole & $1217 \mathrm{cP}$ & 51 & $63,7 \%$ \\
& Yogurt batido sin lactosa de damasco Colun & $1315 \mathrm{cP}$ & 49 & $61,2 \%$ \\
\hline \multirow{4}{*}{ Pudín } & Yogurt batido de piña Soprole & $1774 \mathrm{cP}$ & 31 & $38,7 \%$ \\
& Compota de pera El Vergel & $1899 \mathrm{cP}$ & 69 & $86,2 \%$ \\
& Chiquitín de plátano Nestlé & $2104 \mathrm{cP}$ & 61 & $76,2 \%$ \\
& Yogurt batido natural light endulzado Colún & $2216 \mathrm{cP}$ & 34 & $42,5 \%$ \\
& Flan de chocolate Nestlé & $2272 \mathrm{cP}$ & 79 & $98,7 \%$ \\
\hline
\end{tabular}

\section{Discusión}

A continuación, se analizan los resultados obtenidos en este estudio en comparación con la literatura e investigaciones internacionales realizadas acerca de la viscosidad de los alimentos líquidos utilizados en la intervención de personas con disfagia.

Al realizar una caracterización de los fonoaudiólogos participantes en el estudio, fue posible advertir que un número minoritario de ellos 
$(12,5 \%)$ presentó un tiempo de ejercicio profesional superior a 10 años. Lo anterior puede relacionarse con lo novel que resulta aún el desempeño del fonoaudiólogo en esta área. Respecto del nivel de perfeccionamiento en el ámbito de trastornos de la deglución, ningún sujeto poseía un nivel alto, aspecto que puede explicarse debido a que en Chile no existe una amplia gama de programas de perfeccionamiento en trastornos de la deglución, por lo que el acceso a estos es limitado.

En cuanto al nivel de efectividad total de los fonoaudiólogos, a partir del promedio de aciertos a nivel grupal $(8,02)$, se determinó que alcanzaron un nivel de efectividad regular. No obstante, a nivel individual, gran parte de los sujetos (55\%) presentó un nivel de efectividad suficiente. La discrepancia entre los desempeños a nivel grupal e individual se explica por el bajo número de aciertos logrados por los sujetos con nivel de efectividad insuficiente, lo que produjo un descenso en el promedio de aciertos grupal.

Respecto de la repetibilidad en el nivel de efectividad para la determinación del grado de viscosidad, los fonoaudiólogos fueron consistentes en sus respuestas, independientemente del nivel de efectividad que obtuvieron. Lo anterior concuerda con los datos aportados por Glassburn y Deem (1998), quienes observaron que los profesionales presentaron diferencias intersujeto en cuanto a la determinación de las viscosidades, sin embargo, las variaciones intrasujeto fueron más repetibles.

En relación con la misma repetibilidad intrasujeto, esta es una variable que depende de la metodología utilizada para determinar la viscosidad de los alimentos líquidos, la cual debiese permitir que las respuestas sean constantes en el tiempo, independientemente del acierto. En este sentido, el $97,5 \%$ de los sujetos participantes obtuvo un porcentaje de repetibilidad superior al 50\%, lo cual indicó que la metodología utilizada fue adecuada para este parámetro, pues no produjo variabilidad en las respuestas.

En el marco de las ideas recién planteadas, cabe destacar que en este estudio un profesional obtuvo una repetibilidad inferior al $50 \%$ y un nivel de efectividad suficiente, a partir de lo cual se logra inferir que sus dificultades se enmarcaron en la metodología empleada para determinar la viscosidad. En contraste con lo anterior, hubo dos profesionales que presentaron repetibilidad superior al $50 \%$ y un nivel de efectividad insuficiente, lo que podría vincularse con dificultades en el manejo de conocimientos teóricos acerca del tema.

En relación con el nivel de efectividad y tiempo de ejercicio profesional, se observó que a medida que aumenta el periodo laboral en el área, aumenta la cantidad de sujetos que alcanzan un nivel de efectividad suficiente, por lo que se espera que el tiempo de ejercicio profesional sea una variable que influya en el nivel de efectividad. Sin embargo, no es posible generalizar esta aseveración considerando el bajo número de sujetos con mayor experiencia laboral de este estudio, aspecto que se debería resguardar en futuras investigaciones.

En cuanto al nivel de efectividad $y$ perfeccionamiento, se evidenció que la mayor parte de los fonoaudiólogos (82,5\%) del estudio poseía un bajo nivel de perfeccionamiento, pese a que su nivel de efectividad fue suficiente. En consecuencia, se plantea que este nivel no se rige probablemente por 
conocimientos teóricos adquiridos a través del perfeccionamiento en el área.

Por otra parte, la competencia de los sujetos en la determinación del grado de viscosidad fue mayor en la categoría líquido fino (80\%), seguida por la categoría pudín $(70,2 \%)$. Lo anterior puede estar asociado al hecho de que ambas se encuentran en los extremos inferior y superior, respectivamente, de la clasificación propuesta por Penman y Thomson (Atherton et al., 2007), por lo que la probabilidad de confundirlas con otras categorías es menor. Además, en el caso de líquido fino el alto porcentaje de competencia pudo verse influenciado por el hecho de haber presentado solo un producto perteneciente a esta categoría.

Por el contrario, el menor porcentaje de competencia se evidenció en las categorías néctar $(56,8 \%)$ y miel $(62,5 \%)$, lo cual puede justificarse porque ambas se encuentran en los valores intermedios de la clasificación de Penman y Thomson (Atherton et al., 2007) y, por lo tanto, existe una mayor probabilidad de desacierto para identificar y diferenciar límites entre ellas.

En cuanto a la competencia grupal por producto, se observaron diferencias en el reconocimiento de las seis muestras proporcionadas en la categoría pudín, encontrando un porcentaje de competencia mayor en los productos Flan de chocolate Nestlé y Compota de pera El Vergel. En cambio, los productos Yogurt de piña Soprole y Yogurt natural Colun light presentaron el menor porcentaje de acierto. Esto podría explicarse por los valores de viscosidad objetiva de dichos productos, donde los de mayor competencia se encuentran en el extremo superior de la categoría pudín, mientras que los de menor competencia tienen valores cercanos al límite entre la categoría pudín y miel, lo que pudo generar mayores errores en su reconocimiento.

En el presente estudio se evidenció que no existe una estandarización respecto de los criterios de clasificación empleados para determinar el tipo de viscosidad de los alimentos líquidos, ya que estos variaban según el lugar en el que los fonoaudiólogos se desempeñan laboralmente. Así, por ejemplo, en ciertos recintos solo consideraban el agua y/o sus variantes saborizadas dentro de la categoría líquido fino. Lo anterior se condice con las aseveraciones de Paik y colaboradores (2004), quienes indican que los programas de dietas preparados sobre la base de la definición de la viscosidad de los alimentos carecen de un medio estándar para dicha definición.

Tal como ya se ha mencionado, la variabilidad en el rendimiento de los sujetos en el estudio pudo estar influenciada por la metodología utilizada por ellos, dado que ciertos profesionales emplearon un método de degustación. Esta metodología pudo haber incidido en la determinación del grado de viscosidad debido a que, como señalan Hanson et al. (2012), los alimentos líquidos se comportan de manera distinta una vez que han ingresado a la cavidad oral, pues la saliva inicia un proceso químico y mecánico de ruptura de las estructuras de los alimentos, modificando las características del bolo.

A partir de lo expuesto, considerando el nivel de efectividad regular y alta repetibilidad alcanzada por el grupo, se encontró que los fonoaudiólogos poseen un dominio relativo para determinar la viscosidad de los alimentos líquidos. Esta competencia, sin embargo, no es homogénea entre los sujetos dado que aún existen profesionales que no presentan un 
nivel de efectividad suficiente, lo cual puede explicarse por las diferencias en el manejo de conocimientos, criterios de clasificación y metodologías utilizadas.

\section{Conclusión}

A partir del análisis de los resultados de esta investigación, se pueden establecer las conclusiones que se exponen a continuación. Los profesionales fonoaudiólogos estudiados poseen un dominio relativo para determinar el grado de viscosidad de los alimentos líquidos, no obstante este no es homogéneo, por lo cual es necesario fomentar el conocimiento y un manejo adecuado en el tema. Lo anterior implica generar un lenguaje común que permita consensuar procedimientos y criterios para determinar, de manera óptima, el grado de viscosidad de los alimentos utilizados durante la intervención de personas con disfagia, con el fin de satisfacer sus necesidades y favorecer su calidad de vida.

Los hallazgos de esta investigación sugieren que el dominio para determinar el grado de viscosidad se adquiere a través de conocimientos teóricos y prácticos, por lo que sería recomendable adecuar los cursos que abordan trastornos de la deglución en las mallas curriculares de pregrado de la carrera de Fonoaudiología. Ello, con el objetivo de que las futuras generaciones egresen teniendo los conocimientos necesarios para alcanzar un mejor desempeño en la determinación de la viscosidad. En este mismo sentido, para aquellos sujetos ya egresados, se recomienda incrementar la participación en programas de perfeccionamiento y especialización en el área.
Los conocimientos teóricos y prácticos que requieren los fonoaudiólogos deben centrarse en el desarrollo de habilidades para reconocer de manera óptima las distintas viscosidades, poniendo énfasis en aquellas categorías que en este estudio presentaron mayor dificultad (néctar y miel), y que se ubican en los valores centrales de la clasificación. Lo anterior permitiría disminuir el riesgo de error cuando se determina la viscosidad de forma subjetiva, principalmente en productos de fácil acceso comercial y así poder indicarlos a aquellos usuarios que se encuentran con tratamiento ambulatorio.

En cuanto a otras proyecciones de este trabajo, en el futuro se podría realizar el mismo tipo de investigación incluyendo los productos que se utilizan diariamente en el manejo de usuarios con disfagia. Además, sería recomendable replicar la investigación con profesionales dedicados a la nutrición y dietética, ya que la indicación de la dieta para un usuario con disfagia es un trabajo que se realiza por fonoaudiólogos y nutricionistas en conjunto.

Finalmente, considerando que la información entregada corresponde al dominio de profesionales fonoaudiólogos de la Región Metropolitana de Santiago de Chile, resulta necesario ampliar la investigación a nivel nacional para abarcar un universo mayor de profesionales, que permita comenzar a establecer una estandarización. El equipo de investigación ya inició las gestiones pertinentes para continuar el estudio esta vez corrigiendo las limitaciones presentes en este trabajo como la falta de control en la cantidad de muestras presentadas para cada categoría de viscosidad y en 
la caracterización de los sujetos participantes, según el tiempo de ejercicio profesional y el nivel de perfeccionamiento en el área de trastornos de la deglución.

\section{Referencias}

Atherton, M., Bellis-Smith, N., Cichero, J., \& Suter, M. (2007). Texture-modified foods and thickened fluids as used for individuals with dysphagia: Australian standardised labels and definitions. Nutrition \& Dietetics, 64(s2), 56-76. http://dx.doi.org/10.1111/j.1747$\underline{0080.2007 .00153 . x}$

Clavé, P., De Kraa, M., Arreola, V., Girvent, M., Farrè, R., Palomera, E. \& Serra-Prat, M. (2006). The effect of bolus viscosity on swallowing function in neurogenic dysphagia. Alimentary Pharmacology \& Therapeutics, 24(9)1385-1394. http://dx.doi.org/10.1111/j.1365-2036.2006.03118.x

Clavé, P., Arreola, V., Romea, M., Medina, L., Palomera, E. \& Serrat-Prat, M. (2008). Accuracy of the volume-viscosity swallow test for clinical screening of oropharyngeal dysphagia and aspiration. Clinical Nutrition, 27(6), 806-815. http://dx.doi.org/10.1016/j.clnu.2008.06.011

Ekberg, O. (Ed.) (2012). Dysphagia: Diagnosis and treatment. Malmö: Springer.

Glassburn, D. \& Deem, J. (1998). Thickener viscosity in dysphagia management: Variability among. Dysphagia, 13(4), 218-222. http://dx.doi.org/10.1007/pl00009575

González, R. y Bevilacqua, J. (2009). Disfagia en el paciente neurológico. Revista Hospital Clínico Universidad de Chile, 20(3), 252-262.

Goulding, R. \& Bakheit, A. (2000). Evaluation of the benefits of monitoring fluid thickness in the dietary management of dysphagic stroke patients. Clinical Rehabilitation, 14(2), 119-124. http://dx.doi.org/10.1191/026921500667340586

Hanson, B., O' Leary, M., \& Smith, C. (2012). The effect of saliva on the viscosity of thickened Drinks. Dysphagia, 27(1), 10-19. http://dx.doi.org/10.1007/s00455-011-9330-8
Logemann, J. (1998). Evaluation and treatment of swallowing disorders. Austin, Texas: PRO.ED.

Matsuo, K. \& Palmer, J. (2009). Coordination of mastication, swallowing and breathing. Japanese Dental Science Review, 45(1), 31-40. http://dx.doi.org/10.1016/j.jdsr.2009.03.004

Nazar, G., Ortega, A., Godoy, A., Godoy, J. y Fuentealba, I. (2008). Evaluación fibroscópica de la deglución. Revista de Otorrinolaringología y Cirugía de Cabeza y Cuello, 68(2), 131-142. http://dx.doi.org/10.4067/s0718$\underline{48162008000200004}$

National Dysphagia Task Force, American Dietetic Association. (2002). National dysphagia diet: Standardization for optimal care. EE.UU.: Autor.

O'Leary, M., Hanson, B., \& Smith, C. (2010). Viscosity and non-Newtonian features of thickened fluids used for dysphagia therapy. Journal of Food Science, 75(6), 330-338. http://dx.doi.org/10.1111/j.1750-3841.2010.01673.x

Paik, N., Han, T., Park, J., Lee, E., Park, M., \& Hwang, I. (2004). Categorization of dysphagia diets with the line spread test. Archives of Physical Medicine and Rehabilitation, 85(5), 857-861. http://dx.doi.org/10.1016/i.apmr.2003.08.079

Penman J. P. \& Thomson M. (1998). A review of the textured diets developed for the management of dysphagia. Journal of Human Nutrition and Dietetics, 11(1), 51-60. $\quad$ http://dx.doi.org/10.1046/j.1365277x.1998.00079.x

Perry, L. \& Love, C. (2001). Screening for dysphagia and aspiration in acute stroke: A systematic review. Dysphagia, 16(1), 7-18. http://dx.doi.org/10.1007/pl00021290

Steele, C., Goff, H., \& Van Lieshout, P. (2003). The rheology of liquids: a comparison of clinicians' subjective impressions and objective measurement. Dysphagia, 18(3), 95-182. http://dx.doi.org/10.1007/s00455-002-0104-1

Tobar, R. (2010). Disfagia Orofaríngea: Consideraciones Clínicas en la Alimentación. Revista Colegio de Nutricionistas, 6(11), 13-17. 